\title{
La comprensión lectora en estudiantes del nivel primario de la Institución Educativa $\mathrm{N}^{\circ}$ 64808, Ucayali \\ Reading comprehension in students of the primary level of the Educational Institution $N^{\circ} 64808$, Ucayali
}

Lida Maribel Cosme Solano a lida_maribel@yahoo.com

Michael Paredes Lozano ${ }^{\mathrm{b}}$ mselvatico@ hotmail.com

Ángel Héctor Gómez Landeo ${ }^{\mathrm{c}}$ arzapay@ hotmail.com

\footnotetext{
a Universidad Nacional Intercultural de la Amazonía

${ }^{\mathrm{b}}$ Universidad Nacional Intercultural de la Amazonía

${ }^{c}$ Universidad Nacional de Ucayali
}

Recibido: Enero/ 06/2020 • Aceptado: Abril/02/2020 • Publicado: Abril/30/2020

\section{RESUMEN}

Frente al desconocimiento del nivel de dominio de compresión lectora en diversos textos en lengua materna por los estudiantes del nivel primario en sectores rurales, la interrogante que se respondió en esta investigación fue ¿Cuál es el nivel de logro de la competencia: Lee diversos tipos de texto en su lengua materna en los estudiantes de la Institución Educativa №64808 del distrito de Curimaná?

La investigación fue de tipo básico, nivel descriptivo y tuvo como métodos al científico y descriptivo, en consecuencia, el diseño fue descriptivo simple y la población muestral fue de 43 estudiantes del tercer ciclo de la referida Institución Educativa.

La conclusión fue que el nivel de logro de la competencia: Lee diversos tipos de texto en su lengua materna en los estudiantes de la Institución Educativa $\mathrm{N}^{\circ} 64808$, se encuentra en proceso de aprendizaje.

Palabras clave: Competencia: lee diversos tipos de textos.
ABSTRACT

Faced with the lack of knowledge of the level of reading comprehension in various texts in the mother tongue by students at the primary level in rural areas, the question answered in this research was What is the level of achievement of the competence: Read different types of text in their mother tongue in the students of the Educational Institution $N^{\circ} 64808$ of the Curimaná district?

The research was basic, descriptive level, and had scientific and descriptive methods. Consequently, the design was simple descriptive and the sample population was 43 students from the third cycle of the mentioned Educational Institution.

The conclusion was that the level of achievement of the competence: Read various types of text in their mother tongue in the students of Educational Institution No. 64808, is in the process of learning.

Keywords: Competition: read various types of texts..

${ }^{\mathrm{a}}$ Lida Maribel Cosme-Solano, ${ }^{\mathrm{b}}$ Michael Paredes-Lozano y ${ }^{\mathrm{c}}$ Ángel Héctor Gómez-Landeo Articulo Protegido por Licencia Creative Commons: BY-NC-ND / Protected by Creative Commons: BY-NC-ND. Sendas es una revista de acceso abierto / Sendas is an Open Access Journal. 


\section{INTRODUCCIÓN}

La lectura constituye una de las actividades fundamentales en el ser humano porque a través de ella se comprende a sí mismo y a su entorno, y el Ministerio de Educación promueve su fortalecimiento a través de la competencia: Lee diversos tipos de texto en su lengua materna, cuya práctica se fortalece con un constante diagnóstico, los cuales brindan información oportuna, en primer lugar, para que los estudiantes tengan conciencia del nivel en que se encuentran y, en segundo lugar, para que los demás miembros de la comunidad educativa implementen las medidas correctivas necesarias y oportunas.

En sectores rurales como el Caserío de Las Mercedes en el distrito de Curimaná de la Provincia de Padre Abad en la región Ucayali, se desconoce el nivel de dominio de la lectura, como en el caso de la institución educativa $\mathrm{N}^{\circ} 64808$, cuya comunidad educativa desconocía el nivel de dominio de la competencia: Lee diversos tipos de texto en su lengua materna, por ello, el objetivo de esta investigación fue determinar el nivel de logro de esta competencia.

Al respecto, Llorens (2015), en la investigación: La comprension lectora en educacion primaria: importancia e influencia en los resultados académicos, de la universidad internacional de Rioja. España. Concluyó que alcanzar una buena comprension lectora es uno de los principales retos educativos en las escuelas, ya que de ellas dependerá la calidad del aprendizaje de todas las áreas educativas y el éxito personal social y profesional futuro de los alumnos.

Así mismo, Flores (2018), en la investigación: Gestión curricular en la competencia lee diversos tipos de textos escritos en su lengua materna de la institucion educativa pública $N^{\circ} 16449$ de la universidad San Ignacio de Loyola. Concluyó que el diagnostico sobre el nivel de comprensión de lectura en los estudiantes condujo a la reflexión sobre la limitada gestión curricular en la competencia: lee diversos textos en lengua materna, por parte de los docentes.

Tirado (2018), en la investigación: Monitoreo, Acompañamiento y evaluación para mejorar la práctica docente en la competencia lee diversos tipos de textos en su lengua materna del área de comunicación de los estudiantes del III ciclo de educación básica

${ }^{\mathrm{a}}$ Lida Maribel Cosme-Solano, ${ }^{\mathrm{b}}$ Michael Paredes-Lozano y ${ }^{\mathrm{c}}$ Ángel Héctor Gómez-Landeo Articulo Protegido por Licencia Creative Commons: BY-NC-ND / Protected by Creative Commons: BY-NC-ND. Sendas es una revista de acceso abierto / Sendas is an Open Access Journal. 
regular de la institución educativa N80755 "El carmelo" - distrito de Virú - La Libertad. Concluyó que es menester el diagnóstico debidamente contextualizado para identificar los aspectos que limitan el aprendizaje significativo de los estudiantes y el plan de monitoreo es una herramienta que coadyuva a formar compromisos comune para la toma correcta de decisiones.

Panduro (2017), en la investigación: Estrategía de aprendizaje y la comprensión lectora en estudiantes de educación primaria de la institución educativa $N^{\circ} 65299$ Nueva Libertad del distrito de calleria de Ucayali, de la universidad Católica los Ángeles de Chimbote. Ucayali, Concluyó que las estrategias de aprendizaje en la adquisición de información, codificación y recuperación de información se relacionan significativamente con la comprensión lectora en los estudiantes de educación primaria de la Institución Educativa N ${ }^{\circ} 65229$ Nueva Libertad, del Distrito de Callería, Ucayali, 2017, este diagnóstico indica que el éxito o fracazo de la comprensión lectora depende del tipo de estrategias que practiquen los docentes.

El currículo nacional (2016), a través de la competencia lee diversos tipos de textos en lengua materna, promueve en el estudiante la compresión lectora en si misma con relación a su entorno.

De igual forma el estudiante toma conciencia de los propositos de los diversos tipos de textos y del uso de las mismas en sus diferentes contextos y ámbitos de la vida cotidiana y del papel de la experiencia literaria en la formación de lectores y de las relaciones intertextuales que se establecen entre los textos leídos. Esto es crucial en un mundo donde las nuevas tecnologías y la multimodalidad han transformado los modos de leer. (Ministerio de Educación, 2016).

Según Ministerio de Educación (2016), la competencia lee diversos tipos de textos en lengua materna, durante el tercer ciclo, implica el desarrollo de las siguientes capacidades:

${ }^{\mathrm{a}}$ Lida Maribel Cosme-Solano, ${ }^{\mathrm{b}}$ Michael Paredes-Lozano y ${ }^{\mathrm{c}}$ Ángel Héctor Gómez-Landeo Articulo Protegido por Licencia Creative Commons: BY-NC-ND / Protected by Creative Commons: BY-NC-ND. Sendas es una revista de acceso abierto / Sendas is an Open Access Journal. 
1. Capacidad. Obtiene información del texto escrito: el estudiante localiza y selecciona y diferencia la información que se encuentran en distintas partes de un texto, de igual forma describe el significado de las palabras comparando su entorno con el de otros, para identificar un propósito y realaciones de causa- efecto.

2. Infiere e interpreta información del texto: el estudiante construye el sentido del texto, es decir el estudiante indaga las relaciones entre la información explícita e implicita para deducir una nueva información o completar ciertos vacíos del texto escrito, así como los recursos textuales, para construir el sentido global y profundo del texto, y explicar el propósito, el uso estético del lenguaje, las intenciones del autor, así como la relación con el contexto sociocultural del lector y del texto.

3. Reflexiona y evalúa la forma, el contenido y contexto del texto: los procesos de reflexión y evaluación están relacionados porque ambos suponen que el estudiante se distancie de los textos escritos situados en épocas y lugares distintos, y que son presentados en diferentes soportes y formatos.

Para ello, compara y contrasta aspectos formales y de contenido del texto con la experiencia, el conocimiento formal del lector y diversas fuentes de información. Asimismo, emite una opinión personal sobre aspectos formales, estéticos, contenidos de los textos considerando los efectos que producen, la relación con otros textos, y el contexto sociocultural del texto y del lector.

\section{MÉTODOS}

El tipo de investigación fue no experimental, descriptiva, de tipo básica, de amplitud macroeducativa, de enfoque cuantitativo; como método general se utilizó el científico y como métodos específicos el descriptivo y el estadístico.

Según Hernández et al (2010), el diseño de la investigación se define como una estructura o una organización esquematizada que adopta el investigador para relacionar y controlar las variables de estudio. Para esta investigación, el diseño es el descriptivo simple y su esquema es el siguiente:

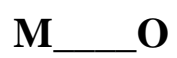
Dónde:
M: Muestra
O: Observación.

${ }^{\mathrm{a}}$ Lida Maribel Cosme-Solano, ${ }^{\mathrm{b}}$ Michael Paredes-Lozano y ${ }^{\mathrm{c}}$ Ángel Héctor Gómez-Landeo Articulo Protegido por Licencia Creative Commons: BY-NC-ND / Protected by Creative Commons: BY-NC-ND. Sendas es una revista de acceso abierto / Sendas is an Open Access Journal. 
La población muestral estuvo constituida por 43 estudiantes de la Institución Educativa №64808 del Centro Poblado Las Mercedes del Distrito de Curimaná, se utilizó la técnica de prueba pedagógica, El instrumento que se utilizó fue la prueba escrita o test de comprensión de lectura.

\section{RESULTADOS}

Tabla 1. Resultado del nivel de logro de la competencia: lee diversos tipos de texto en su lengua materna

\begin{tabular}{lcc}
\hline \multicolumn{1}{c}{ Nivel } & Frecuencia & Porcentaje \\
\hline Logro destacado (18-20) & 2 & 5 \\
Logro previsto (14-17) & 9 & 21 \\
En proceso (11-13) & 17 & 39 \\
Inicio (0-10) & 15 & 35 \\
Total & 43 & 100.00 \\
\hline
\end{tabular}

Fuente: Base de datos (Excel) obtenidos del instrumento de investigación.

Como se puede apreciar, de los 43 estudiantes que representa el 100\%, el 39\% se encuentra en el nivel de proceso; mientras que el 35\% se encuentra en el nivel de inicio, el $21 \%$, se encuentra en logro previsto y una minoría de $5 \%$, se encuentra en logro destacado. En síntesis, el nivel de logro de esta competencia se encuentra en proceso de aprendizaje.

\section{Tabla 2. Resultado de la dimensión, capacidad: Obtiene información del texto escrito}

\begin{tabular}{lcc}
\hline Nivel & Frecuencia & Porcentaje \\
\hline Logro destacado (18-20) & 15 & 35 \\
Logro previsto (14-17) & 8 & 19 \\
En proceso (11-13) & 10 & 23 \\
Inicio (0-10) & 10 & 23 \\
Total & 43 & 100.00 \\
\hline
\end{tabular}

Fuente: Base de datos (Excel) obtenidos del instrumento de investigación.

Como se puede apreciar, de los 43 estudiantes que representa el 100\%, la gran mayoría, $35 \%$, se encuentra en el nivel de logro destacado; mientras que un $23, \%$, se encuentra en el nivel de proceso y otro $23 \%$ se encuentra en el nivel de inicio, mientras una minoría de $19 \%$ se encuentran en logro previsto. En síntesis, el logro de esta capacidad se encuentra en nivel destacado.

${ }^{\mathrm{a}}$ Lida Maribel Cosme-Solano, ${ }^{\mathrm{b}}$ Michael Paredes-Lozano y ${ }^{\mathrm{c}}$ Ángel Héctor Gómez-Landeo Articulo Protegido por Licencia Creative Commons: BY-NC-ND / Protected by Creative Commons: BY-NC-ND. Sendas es una revista de acceso abierto / Sendas is an Open Access Journal. 
Tabla 3. Resultado de la dimensión: capacidad: infiere e interpreta información del texto escrito

\begin{tabular}{lccc}
\hline & Nivel & Frecuencia & Porcentaje \\
\hline Logro destacado (18-20) & 2 & 4 \\
Logro previsto (14-17) & 9 & 21 \\
En proceso (11-13) & 27 & 63 \\
Inicio (0-10) & 5 & 12 \\
Total & 43 & 100.00 \\
\hline
\end{tabular}

Fuente: Base de datos (Excel) obtenidos del instrumento de investigación.

Como se puede apreciar, de los 43 estudiantes que representa el 100\%, la gran mayoría que viene a ser el $63 \%$, se encuentra en proceso; mientras que el $21 \%$, se encuentra en logro previsto, el $12 \%$ obtuvo un nivel de logro en inicio y una minoría de $4 \%$, logro destacado. En síntesis, el nivel de logro de esta capacidad se encuentra en proceso de aprendizaje.

Tabla 4. Resultado de las dimensiones: capacidad: reflexiona y evalúa la forma, el contenido y el contexto del texto escrito

\begin{tabular}{lcc}
\hline \multicolumn{1}{c}{ Nivel } & Frecuencia & Porcentaje \\
\hline Logro destacado (18-20) & 0 & 0.0 \\
Logro previsto (14-17) & 7 & 16 \\
En proceso (11-13) & 24 & 56 \\
Inicio (0-10) & 12 & 28 \\
Total & 43 & 100.00 \\
\hline
\end{tabular}

Fuente: Base de datos (Excel) obtenidos del instrumento de investigación.

Como se puede apreciar, de los 43 estudiantes que representa el 100\%, la gran mayoría que viene a ser el 56\%, se encuentra en el nivel de proceso; mientras que el 28\%, se encuentra en inicio, el 16\%, se encuentra el nivel de logro previsto y ningún estudiante se encuentra en logro destacado. En síntesis, el nivel de logro de esta capacidad se encuentra en proceso de aprendizaje.

\section{DISCUSIÓN}

El resultado de la competencia: Lee diversos tipos de texto en su lengua materna de los estudiantes del tercer ciclo de la Institución Educativa $\mathrm{N}^{\circ} 64808$, se encuentra en proceso

${ }^{\mathrm{a}}$ Lida Maribel Cosme-Solano, ${ }^{\mathrm{b}}$ Michael Paredes-Lozano y ${ }^{\mathrm{c}}$ Ángel Héctor Gómez-Landeo Articulo Protegido por Licencia Creative Commons: BY-NC-ND / Protected by Creative Commons: BY-NC-ND. Sendas es una revista de acceso abierto / Sendas is an Open Access Journal. 
de aprendizaje, sin embargo, existe una variación de resultados con respecto a las dimensiones o capacidades, la primera capacidad: obtiene información del texto escrito, se encuentra en logro destacado, mientras que la segunda capacidad: infiere e interpreta información del texto escrito, se encuentra en proceso de aprendizaje y la última capacidad: reflexiona y evalúa la forma, el contenido y el contexto del texto escrito, se encuentra en proceso de aprendizaje.

Al respecto, el Ministerio de Educación en el Currículo Nacional (2016) define esta competencia como una interacción dinámica entre el lector, el texto y los contextos socioculturales que enmarcan la lectura. Los hallazgos, demuestran la predisposición y el potencial de los estudiantes con respecto a la primera capacidad: Obtiene información del texto escrito, sin embargo, existe serias limitaciones con respecto a las capacidades de inferencia a interpretación, así como de la capacidad de reflexión y evaluación de textos escritos.

Tirado (2018) resalta lo obtenido en el diagnóstico, como identificar los problemas que afectan el logro de aprendizajes de los estudiantes, en un ambiente en donde la comunidad educativa se siente responsable, creándose a partir de ello nuevos instrumentos de diagnóstico con miras a coadyuvar en el éxito escolar de los estudiantes y de los demás integrantes de la comunidad educativa.

Asimismo, el Ministerio de Educación en el curriculo nacional (2016) sostiene que al involucrar al estudiante con la lectura, éste contribuye con su desarrollo personal, así como el de su propia comunidad, además de conocer e interactuar con contextos socioculturales distintos del suyo. Los hallazgos indican que la relacion de los estudiantes con la lectura se encuentra en proceso, es decir aun no logran involucrar a los estudiantes con la lectura, y el tercer ciclo es un periodo oportuno para desarrollarlo, con la finalidad de aportar en el crecimiento personal y social del estudiante.

El resultado guarda relación con los de Panduro (2017), quien refiere que el diagnóstico de la comptencia Lee diversos tipos de texto en su lengua materna, se debe hacer por etapas,

${ }^{\mathrm{a}}$ Lida Maribel Cosme-Solano, ${ }^{\mathrm{b}}$ Michael Paredes-Lozano y ${ }^{\mathrm{c}}$ Ángel Héctor Gómez-Landeo Articulo Protegido por Licencia Creative Commons: BY-NC-ND / Protected by Creative Commons: BY-NC-ND. Sendas es una revista de acceso abierto / Sendas is an Open Access Journal. 
respetando las particularidades de cada capacidad como el de Obtiene información del texto escrito, asimismo las estrategias se deben realizar por capacidades, pues el dominio de una, garantizará el dominio de la siguiente, para que el aprendizaje de la competencia sea sostenible en el tiempo.

Al respecto el Ministerio de Educación (2016) sostiene que la capacidad: Obtiene información del texto escrito, se refiere a que el estudiante localiza y selecciona información explícita en textos escritos con un propósito específico. Los hallazgos indican que para el estudiante no resulta complicado el desarrollo de esta capacidad, pues coadyuva en el fortalecimiento de otras capacidades como el razonamiento.

Por su parte, Tangoa \& Hidalgo, (2016) señalan que la comprensión lectora se relaciona con el estilo de aprendizaje. Al respecto el Ministerio de Educación (2016) nos dice que el estudiante esta en la capacidad de construir el texto, establece relaciones entre la información explícita e implícita, para deducir una nueva información o completar los vacíos del texto escrito. Los hallazgos indican que a pesar que el estudiante pueda desarrollar una de las capacidades, esto no es garantía de que logre desarrollar todas las capacidades con respecto a la lectura, es así que la capacidad de inferencia e interpretacion del texto requiere de otras estrategias que garanticen su desarrollo, por lo que se hace menester su constante diagnóstico para identificar las fortalezas y respectivas debilidades.

Añade esta entidad que el estudiante se relaciona con el contenido del texto y genera deducciones entre la información implícita y la información explícita, así como los recursos textuales, para construir el sentido global y profundo del texto, y explicar el propósito, el uso estético del lenguaje, las intenciones del autor, así como la relación con el contexto sociocultural del lector y del texto. Los hallazgos indican que los estudiantes no realizan las mencioandas competencias, por lo que es importante la implementacion de un plan de mejora.

Asimismo, Flores (2018) resalta la importancia del diagnóstico oportuno, porque permite encontrar las causas que conlleva a los estudiantes a tener problemas de comprensión

${ }^{\mathrm{a}}$ Lida Maribel Cosme-Solano, ${ }^{\mathrm{b}}$ Michael Paredes-Lozano y ${ }^{\mathrm{c}}$ Ángel Héctor Gómez-Landeo Articulo Protegido por Licencia Creative Commons: BY-NC-ND / Protected by Creative Commons: BY-NC-ND. Sendas es una revista de acceso abierto / Sendas is an Open Access Journal. 
lectora, como el factor docente, con la finalidad de fortalecerlo en el dominio de la competencia Lee diversos tipos de texto en su lengua materna, así como en la obtención del texto, inferencia e interpretación y reflexión del mismo para que pueda asesorar con mayor eficacia a los estudiantes.

Al respecto, el Ministerio de Educación (2016) manifiesta que los procesos de reflexión y evaluación están relacionados porque ambos suponen que el estudiante se distancie de los textos escritos situados en épocas y lugares distintos, y que son presentados en diferentes soportes y formatos. Los hallazgos demuestran que los estudiantes de tercer ciclo tienen limitaciones con la refexión y evaluación de textos escritos.

Finalmente, se relaciona con Llorens (2015), quien concluyó que una buena comprensión lectora es uno de los principales retos educativos en las escuelas, ya que de ellas dependerá la calidad del aprendizaje de todas las áreas educativas y el éxito personal social y profesional futuro de los estudiantes.

\section{CONCLUSIONES}

1. Se concluye que el nivel de logro de la competencia Lee diversos tipos de texto en su lengua materna de los estudiantes del tercer ciclo de la Institución Educativa $N^{\circ} 64808$ del Caserío las Mercedes de Curimaná, Ucayali 2019 se encuentra en proceso de aprendizaje.

2. Así mismo, en este mismo nivel se encuentran los logros obtenidos en las capacidades Infiere e interpreta información del texto escrito y Reflexiona y evalúa la forma, el contenido y el contexto del texto escrito de estos mismos estudiantes.

3. Sin embargo, los estudiantes del tercer ciclo de la Institución Educativa $\mathrm{N}^{\circ} 64808$ del Caserío las Mercedes de Curimaná, Ucayali 2019 obtuvieron un nivel de logro destacado en la capacidad Obtiene información del texto escrito.

${ }^{\mathrm{a}}$ Lida Maribel Cosme-Solano, ${ }^{\mathrm{b}}$ Michael Paredes-Lozano y ${ }^{\mathrm{c}}$ Ángel Héctor Gómez-Landeo Articulo Protegido por Licencia Creative Commons: BY-NC-ND / Protected by Creative Commons: BY-NC-ND. Sendas es una revista de acceso abierto / Sendas is an Open Access Journal. 


\section{REFERENCIAS}

Flores, W. C. (2018). Gestión curricular en la competencia lee diversos tipos de textos escritos en su lengua materna de la institucion educativa pública $N^{\circ} 16449$. Lima Perú: Universidad San Ignacio de Loyola.

Hernández, R., Fernández, C., \& Baptista, P. (2010). Metodología de la Investigación. Mexico D.F.: Mc Graw Hill.

Jauregui, Y. C. (2018). Comprensión lectora y rendimiento académico en el área de comunicación en los estudiantes de primaria de la institución educativa "Marko Emilio Jara Schenone" Pucallpa - 2017. Pucallpa - Perú: Universidad Nacional de Ucayali.

Llorens, R. (2015). La comprension lectora en educacion primaria: importancia e influencia en lso resultados academicos. España: Universidad Internacional de Rioja.

Ministerio de Educación. (2016). Currículo nacional . Lima: Ministerio de Educación.

Ministerio de Educación. (2016). Curriculo Nacional de la Educación Básica. Lima : MINEDU.

Panduro, N. (2017). Estrategía de aprendizaje y la comprensión lectora en estudiantes de educación primaria de la institución educativa $N^{\circ} 65299$ Nueva Libertad del distrito de calleria de Ucayali, 2017. Pucallpa - Perú: Universidad Católica los Ángeles de Chimbote.

Tangoa, N., \& Hidalgo Mozombite, G. (2016). Estilo de aprendizaje y comprensión lectora de los estudiantes del quinto grado de educación primaria de la institución educativa $N^{\circ} 62313$ de Palmichi del pueblo Shawi Cahuapanas - Loreto 2015. Yarinacocha Perú: Universidad Nacional Intercultural de la Amazonía.

Tirado, M. I. (2018). Monitoreo, Acompañamiento y evaluación para mejorar la práctica docente en la competencia lee diversos tipos de textos en su lengua materna del área

${ }^{\mathrm{a}}$ Lida Maribel Cosme-Solano, ${ }^{\mathrm{b}}$ Michael Paredes-Lozano y ${ }^{\mathrm{c}}$ Ángel Héctor Gómez-Landeo Articulo Protegido por Licencia Creative Commons: BY-NC-ND / Protected by Creative Commons: BY-NC-ND. Sendas es una revista de acceso abierto / Sendas is an Open Access Journal. 
de comunicación de los estudiantes del III ciclo de educación básica regular de la institución educativa $N$. La Libertad - Perú: Instituto pedagógico Nacional Monterrico.

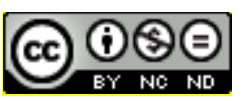

La comprensión lectora en estudiantes del nivel primario de la Institución Educativa $\mathbf{N}^{\circ} \mathbf{6 4 8 0 8}$, Ucayali (Lida Maribel Cosme-Solano) Por Revista Kolpa se encuentra bajo una Licencia Creative Commons-No Comercial-Sin Derivadas 3.0 Uported.

${ }^{\mathrm{a}}$ Lida Maribel Cosme-Solano, ${ }^{\mathrm{b}}$ Michael Paredes-Lozano y ${ }^{\mathrm{c}}$ Ángel Héctor Gómez-Landeo Articulo Protegido por Licencia Creative Commons: BY-NC-ND / Protected by Creative Commons: BY-NC-ND. Sendas es una revista de acceso abierto / Sendas is an Open Access Journal. 